\title{
AUTOMEDICAÇÃO NO PERÍODO DA PANDEMIA COVID-ı
}

\author{
Rosangela Costa Alves ${ }^{1}$ \\ Andrelina Cordeiro ${ }^{2}$ \\ Vinícius Mendes Souza Carneiro ${ }^{3}$
}

RESUMO: A automedicação consiste em administrar por conta própria um ou mais medicamentos sem a orientação profissional e, com a grande quantidade de informações acessíveis na internet tornou- se um hábito comum no período de pandemia do novo coronavírus. O presente trabalho teve como objetivo expor sobre a utilização inadequada de medicamentos prescritos e isentos devido ao seu fácil acesso durante o isolamento social, retratar a possibilidade de efeitos indesejáveis relacionados aos medicamentos, consequente da automedicação, e destacar a ausência da prática clínica e de assistência nos fornecimentos farmacêuticos ao paciente. Os dados foram obtidos entre março de $2020 \mathrm{e}$ setembro de 2021. A Cloroquina/hidroxicloroquina, vitamina $\mathrm{C}$, ivermectina, azitromicina, ibuprofeno e lopinavir-ritonavir são os medicamentos mais citados nos artigos científicos. Os antirretrovirais foram relatados em sinergia com cloroquina/hidroxicloroquina, que foi a mais citada nos artigos relacionados à automedicação. $\mathrm{O}$ uso incorreto dessas substâncias pode causar efeitos colaterais graves, outros tipos de patologias ou até dependência. Sendo assim, o farmacêutico e a equipe multiprofissional devem orientar o paciente sobre o uso racional de medicamentos.

Palavras-chave: Automedicação. Pandemia. Coronavírus.

ABSTRACT: Self-medication consists of self-administering one or more medications without professional guidance and, with the large amount of information accessible on the internet, it has become a common habit during the new coronavirus pandemic period. This study aimed to expose the inappropriate use of prescription and exempt drugs due to their easy access during social isolation, portray the possibility of undesirable effects related to drugs, resulting from self-medication, and highlight the absence of clinical and care practice in pharmaceutical supplies to the patient. Data were obtained between March 2020 and September 2021. Chloroquine/hydroxychloroquine, vitamin C, ivermectin, azithromycin, ibuprofen and lopinavir-ritonavir are the drugs most cited in scientific articles. Antiretrovirals were reported in synergy with chloroquine/hydroxychloroquine, which was the most cited in articles related to self-medication. The incorrect use of these substances can cause serious side effects, other types of pathologies or even addiction.

\footnotetext{
I Curso: Farmácia. Instituição: Universidade Salvador (UNIFACS). E-mail: rosangelaalves2org.I@gmail.com.

${ }^{2}$ Curso: Farmácia. Instituição: Universidade Salvador (UNIFACS) E-mail: cordeiro 77@gmail.com.

3 Orientador. Docente da Universidade Salvador (UNIFACS).E-mail: vinicius.carneiro@unifacs.br
} 
Therefore, the pharmacist and the multidisciplinary team must guide the patient about the rational use of medication.

Keywords: Self-medication. Pandemic. Coronavirus.

\section{INTRODUÇÃO}

De acordo com a Organização Pan Americana de Saúde e Organização Mundial de Saúde (OMS), o surto da doença causada pelo novo coronavírus (COVIDı́) se tornou uma Emergência de Saúde Pública de Importância Internacional em janeiro de 2020. Em março, a COVID-ıg foi caracterizada pela OMS como uma pandemia. Foram confirmados no País 606.726 óbitos e 21.765.420 casos de coronavírus desde o início da pandemia.

A pandemia da doença causada pelo novo coronavírus 2019 (COVID-I9) tornou-se um dos grandes desafios do século XXI. Atualmente ela afeta, mais de roo países e territórios nos cinco continentes. Seus impactos ainda são inestimáveis, mas afetam direta e/ou indiretamente a saúde e a economia da população mundial.

A COVID-ı́ é uma doença infectocontagiosa causada pelo coronavírus da síndrome respiratória aguda grave 2 , do inglês severe acute respiratory syndrome-associated coronavirus (SAER-Cov-2).

O risco de contaminação pela COVID-ı́ é generalizado, mas os recursos apresentados por diferentes grupos sociais para o enfrentamento da situação, fazem com que os mais afetados são os indivíduos de baixa renda. Estes são os que vivem nas periferias, os que não contam com abastecimento de água expostos a condições inadequadas. Proporcionalmente a população negra, a população indígena, entre outros que têm menos acesso aos seus direitos seriam os grupos mais suscetíveis. (PEREIRA, et al., 2020).

Vale ainda ressaltar que no período de pandemia de coronavírus pode haver o aumento de um hábito comum e cultural do brasileiro: o uso indiscriminado de medicamentos. A venda de medicamentos relacionados à Covid-ıg aumentou significativamente no Brasil desde o início da pandemia, de acordo com pesquisa encomendada pelos conselhos de Farmácia, divulgada no final do mês de abril de 2021. Suplementos alimentares, antigripais, vitaminas e minerais, entre outros medicamentos, estão entre os mais procurados na busca para fortalecer a imunidade e prevenir a infecção pelo coronavírus, mesmo sem 
comprovação científica. Visando verificar se houve um aumento no hábito da automedicação foi feito um levantamento bibliográfico sobre o tema.

\section{METODOLOGIA}

Trata-se de uma revisão integrativa da literatura de estudos envolvendo publicações científicas sobre a automedicação no período de pandemia de COVIDı́. A revisão integrativa é um método de revisão mais amplo, pois permite incluir literatura teórica e empírica bem como estudos com diferentes abordagens metodológicas. Os estudos incluídos na revisão são analisados de forma sistemática em relação aos seus objetivos, materiais e métodos, permitindo que o leitor analise o conhecimento pré-existente sobre o tema investigado (Pompeo, Rossi \& Galvão, 2009). Vale ressaltar que foram pesquisados cerca de 30 artigos sendo que só io artigos responderam diretamente ao objetivo da pesquisa.

Sendo assim, a construção induzida a partir do tema a Automedicação no período de pandemia de COVID-ı, resultou na pergunta: Quais os fatores que corroboram para a prática da automedicação em período de pandemia de COVIDıg? Os descritores utilizados na busca foram: “Automedicação”, "Pandemia”, "Corona Vírus”. Para o cruzamento dos descritores em português e inglês. $\mathrm{Na}$ coleta de dados foi realizada a busca de artigos científicos nos bancos de dados, Online Medical Literature Analyses and Retrieval System Online (MEDLINE), Scientific Eletronic Library Online (SCIELO), Medical Publications (PUBMED), Red Iberoamericana de Innovación y Conocimiento Científico (REDIB). Essa busca utilizou as terminologias cadastradas nos Descritores em Ciências da Saúde - DeCs, criados pela Biblioteca Virtual em Saúde (BVS) desenvolvido a partir do Medical Subject Headings (MESH) da U.S. National Library of Medicine, que permite o uso da terminologia comum em português.

\section{DESENVOLVIMENTO}

Após realização de busca mais detalhada sobre os artigos, observa-se à importância da pesquisa sobre o tema explorado, obtendo à um nível maior de informações aos leitores. 


\begin{tabular}{|c|c|c|c|c|}
\hline Autor/Ano & Titulo & Método & Objetivos & Resultados \\
\hline $\begin{array}{l}\text { Pereira, M. S. } \\
(2020)\end{array}$ & $\begin{array}{l}\text { Pandemia: os } \\
\text { riscos da } \\
\text { automedicação } \\
\text { e os cuidados } \\
\text { com a saúde. }\end{array}$ & Pesquisa & $\begin{array}{l}\text { Estimar a } \\
\text { prevalência de } \\
\text { automedicação } \\
\text { para prevenir o } \\
\text { COVID-ı e } \\
\text { seus } \\
\text { fatores } \\
\text { associados } \\
\text { em Lomé, } \\
\text { Togo. }\end{array}$ & $\begin{array}{l}\text { O estudo } \\
\text { confirmou que } \\
\text { a } \\
\text { automedicação } \\
\text { era } \\
\text { motivada } \\
\text { principalmente } \\
\text { para uso } \\
\text { preventivo e } \\
\text { não } \\
\text { para tratamento } \\
\text { de } \\
\text { manifestações } \\
\text { clínicas } \\
\text { específicas de } \\
\text { COVID-I }\end{array}$ \\
\hline $\begin{array}{l}\text { Oliveira, A. G. } \\
\text { L. et al. (2020). }\end{array}$ & $\begin{array}{l}\text { Os riscos da } \\
\text { automedicação } \\
\text { no tratamento } \\
\text { do covid-ı9: } \\
\text { uma revisao de } \\
\text { literatura. }\end{array}$ & Pesquisa & $\begin{array}{l}\text { Objetivo } \\
\text { avaliar a } \\
\text { prevalência de } \\
\text { medicamentos } \\
\text { automedicados } \\
\text { para sintomas } \\
\text { respiratórios, }\end{array}$ & $\begin{array}{l}\text { o durante a } \\
\text { pandemia, o } \\
\text { principal } \\
\text { motivo para o } \\
\text { ato, foi por } \\
\text { relatar que } \\
\text { estava } \\
\text { gripado(a). } \\
\text { Deste } \\
\text { percentual foi } \\
\text { visualizado } \\
\text { uma } \\
\text { porcentagem } \\
\text { relevante, } \\
\text { quando } \\
\text { refere drogas } \\
\text { relacionadas ao } \\
\text { COVID-ı: } \\
\text { como } \\
\text { prevenção, } \\
\text { presença } \\
\text { de sintomas e } \\
\text { caso } \\
\text { confirmado. }\end{array}$ \\
\hline $\begin{array}{l}\text { WANG, M. et } \\
\text { al }\end{array}$ & $\begin{array}{l}\text { O remdesivir e } \\
\text { a cloroquina } \\
\text { inibem }\end{array}$ & Pesquisa & $\begin{array}{l}\text { Avaliar se essa } \\
\text { medicações são } \\
\text { eficaz }\end{array}$ & $\begin{array}{l}\text { Segundo } \\
\text { pesquisa, } \\
\text { nenhum }\end{array}$ \\
\hline
\end{tabular}




\begin{tabular}{|c|c|c|c|c|}
\hline & $\begin{array}{l}\text { efetivamente o } \\
\text { novo } \\
\text { coronavírus } \\
\text { recém-surgido } \\
(2019-\mathrm{nCoV})\end{array}$ & & $\begin{array}{l}\text { notratamento } \\
\text { convid i9 }\end{array}$ & $\begin{array}{l}\text { dois } \\
\text { medicamentos } \\
\text { reduziu o grau } \\
\text { de insuficiência } \\
\text { respiratória ou } \\
\text { inflamações em } \\
\text { pacientes } \\
\text { contaminados } \\
\text { com o } \\
\text { coronavírus }\end{array}$ \\
\hline $\begin{array}{l}\text { SOUZA et. } \\
\text { al., 2021. }\end{array}$ & $\begin{array}{l}\text { Aparición de la } \\
\text { automedicación } \\
\text { en la } \\
\text { población } \\
\text { brasileña } \\
\text { como estrategia } \\
\text { preventiva del } \\
\text { SARSCoV-2. / } \\
\text { Occurrence of } \\
\text { Self-medication } \\
\text { in the } \\
\text { Brazilian } \\
\text { population as } \\
\text { a preventive } \\
\text { strategy for } \\
\text { SARS-CoV-2. } \\
\text { / } \\
\text { Ocorrência de } \\
\text { Automedicação } \\
\text { na } \\
\text { população } \\
\text { Brasileira } \\
\text { como estratégia } \\
\text { preventiva ao } \\
\text { SARSCoV-2 }\end{array}$ & $\begin{array}{l}\text { Estudo } \\
\text { descritivo e } \\
\text { analítico, de } \\
\text { natureza } \\
\text { qualitativa. }\end{array}$ & $\begin{array}{l}\text { Objetivou-se } \\
\text { identificar a } \\
\text { existência de } \\
\text { automedicação } \\
\text { por pessoas } \\
\text { com o } \\
\text { objetivo de } \\
\text { prevenir o } \\
\text { SARSCoV-2 e } \\
\text { analisar } \\
\text { o potencial de } \\
\text { agravamento } \\
\text { desse uso ao } \\
\text { organismo } \\
\text { humano. }\end{array}$ & $\begin{array}{l}30,8 \text { \% } \\
\text { relataram se } \\
\text { automedicar no } \\
\text { intuito de } \\
\text { fortalecer a } \\
\text { imunidade. A de } \\
\text { origem aquição dos } \\
\text { fármacos } \\
\text { constatou cerca } \\
\text { de } \\
\text { 91, 2 o em } \\
\text { farmácia } \\
\text { comercial. }\end{array}$ \\
\hline
\end{tabular}

Um estudo realizado no Brasil constatou que apesar de já existirem normas para combate à compra sem receita, uma grande parte dos usuários, estão esclarecidos sobre a ilegalidade da compra de certos fármacos sem prescrição médica. A prática de ingestão de fármacos sem a prescrição médica, o aconselhamento e o acompanhamento de um profissional de saúde gabaritado se configura como automedicação (Santos, et al., 2018).

A venda de medicamentos relacionados à Covid-ı aumentou significativamente no Brasil desde o início da pandemia, de acordo com pesquisa encomendada pelos conselhos 
de Farmácia, divulgada no final do mês de abril de 2020. Suplementos alimentares, antigripais, vitaminas e minerais, entre outros medicamentos, estão entre os mais procurados na busca para fortalecer a imunidade e prevenir a infecção pelo coronavírus, mesmo sem comprovação científica.

Entre os medicamentos que tiveram aumento nas vendas, conforme a pesquisa, estão Hidroxicloroquina, Paracetamol, Dipirona Sódica, Colecalciferol (Vitamina D) e Ácido Ascórbico (Vitamina C). É importante ressaltar que não temos nada definido cientificamente em relação a medicamentos no combate à Covid-ı9. Atualmente, não existem agentes terapêuticos contra o vírus, e, as pesquisas em desenvolvimento sugerem uma considerável lista de medicamentos com efeitos farmacológicos apropriados e eficácia terapêutica no tratamento de pacientes com a COVID-ı9 (Souza, et al., 2021).

Os tratamentos farmacológicos sugeridos para o tratamento da COVIDı̀ são vários, desde o uso de hidroxicloroquina (HCQ) associado a azitromicina ou em monoterapia, uso de parasitário como a nitazoxanida e outros antivirais tais como lopinavir e ritonavir. Outra alternativa bastante promissora é a terapia com o uso de plasma de pacientes após uma infecção viral. A HCQ é um antimalárico derivado da cloroquina (CQ) com menos preocupações em relação às interações medicamentosas. No entanto é uma das opções de agente farmacológico para o tratamento da COVID-I9 mas ainda sem evidências clínicas de que seja eficaz no tratamento dessa doença (WANG et al., 2020). A automedicação é uma realidade bastante frequente em diversas faixas etárias, bem como em culturas diferentes em que o indivíduo seleciona e escolhe certos medicamentos a fim de tratar um determinado problema de saúde.

Em meio a uma das maiores pandemias da história, os conselhos de Farmácia realizam, a partir de 5 de maio, mais uma campanha sobre a importância do uso racional de medicamentos para a proteção à saúde. A data é alusiva ao tema e neste ano, em que o mundo enfrenta um inimigo poderoso e pouco conhecido. $O$ motivo do alerta é o resultado de um estudo realizado a pedido dos conselhos, pela consultoria IQVIA, que constatou um aumento significativo nas vendas de alguns medicamentos relacionados à Covid-ı nos três primeiros meses desse ano, quando aumentaram os casos da doença, em relação ao mesmo período do ano passado. Veja os dados abaixo: 


\begin{tabular}{|c|c|c|c|}
\hline $\begin{array}{c}\text { MEDICAMENTO/PRINCÍPIO } \\
\text { ATIVO }\end{array}$ & JAN A MAR/2019 & $\begin{array}{l}\text { JAN } \\
\text { MAR/2020 }\end{array}$ & A \\
\hline $\begin{array}{c}\text { HIDROXICLOROQUINA } \\
\text { SULFATO }\end{array}$ & 231.546 & 388.829 & $67,93 \%$ \\
\hline IBUPROFENO & 15.010 .195 & 14.615 .066 & $-2,63 \%$ \\
\hline PARACETAMOL & 11.150 .452 & 19.774 .819 & $77,35 \%$ \\
\hline DIPIRONA SÓDICA & 30.226 .256 & 46.716 .599 & $54,56 \%$ \\
\hline $\begin{array}{c}\text { COLECALCIFEROL } \\
\text { (VITAMINA D) }\end{array}$ & 4.440 .289 & 6.019 .038 & $35,56 \%$ \\
\hline $\begin{array}{c}\text { ACIDO ASCÓRBICO } \\
\text { (VITAMINA C) }\end{array}$ & 9.327 .016 & 26.116 .340 & $180,01 \%$ \\
\hline
\end{tabular}

Fonte: Comunicação do CFF

Os conselhos de Farmácia alertam que todos os medicamentos oferecem riscos. Mesmo os isentos de prescrição podem causar danos, especialmente se forem usados sem indicação ou orientação profissional. Dependendo da dose, o paracetamol pode causar hepatite tóxica. A dipirona oferece risco de choque anafilático e agranulocitose, e o ibuprofeno é relacionado a tonturas e visão turva. Já o uso prolongado da vitamina C pode causar diarreias, cólicas, dor abdominal e dor de cabeça. E com a ingestão excessiva de vitamina $\mathrm{D}$, o cálcio pode depositar-se nos rins e até causar lesões permanentes.

Os riscos são mais graves em relação à hidroxicloroquina, medicamento indicado para tratar doenças como o lúpus eritematoso. Da mesma forma que a cloroquina (indicada para a malária, porém disponibilizada apenas na rede pública), a hidroxicloroquina pode causar problemas na visão, convulsões, insônia, diarreias, vômitos, alergias graves, arritmias e até parada cardíaca. $\mathrm{O}$ uso de hidroxicloroquina ou cloroquina em pacientes internados com teste positivo para o novo coronavírus ainda não tem evidências representativas. Portanto, se justifica apenas com supervisão e prescrição médica, atualmente, com retenção de receita.

\section{CONSIDERAÇÕES FINAIS}

Esse artigo trouxe abordagens amplas $e$ diferentes nas pesquisas sobre a automedicação no período de pandemia de COVID-ı, desde os títulos até a abordagem metodológica. A situação provoca, desde então, preocupantes impactos sociais, econômicos 
e graves consequências para o sistema de saúde global. A automedicação tem sido de grande preocupação aos serviços de saúde, pois, o acesso a assistência médica juntamente com medicamentos, não implica em melhores condições de saúde ou até mesmo, qualidade de vida, pois as falhas na dispensação, o uso de medicamentos por conta própria pode levar a tratamentos que são ineficazes e nada seguros (Oliveira, et al., 2020).

Diante do exposto, o consumo de auto medicação de forma errada pela população aumentou consideravelmente. $\mathrm{O}$ uso irracional de medicamentos tomou frente, por conta dos supostos tratamentos para a Covid-19. Vale ressaltar que, até o momento, não há nenhuma evidência científica conclusiva que possa corroborar com o uso de determinados medicamentos para o tratamento ou profilaxia contra o coronavírus. Logo, posteriores trabalhos, perante esse tema deverão levar em consideração os mais diversos problemas que a automedicação pode vir a trazer, bem como os riscos da ingestão indiscriminada de antibióticos e das possíveis interações medicamentosas que podem vir a ocorrer, é importante salientar o cuidado com o processo de medicação e a filtragem de informações divulgadas pela mídia frente aos cuidados que devem ser realizados.

\section{REFERÊNCIAS}

Conselho Federal de Farmácia - Brasil - Notícia: 30/04/2020 - Levantamento mostra como o medo da COVID-ı impactou venda de medicamentos [Internet]. Cff.org.br. 2020 [cited 27 May 2020]. Available from: http://www.cff.org.br/noticia.php?id=5747

Oliveira, A. G. L. et al. (2020). Os riscos da automedicação no tratamento do covid19: uma revisao de literatura. Centro Universitário Católica de Quixadá. 4200-129641PB.pdf.

Pompeo, D. A; Rossi, L. A; Galvao, C. M. (2009). Revisão integrativa: etapa inicial do processo de validação de diagnóstico de enfermagem. Acta Paul. Enferm. 22(4), 434438.

Pereira, M. S. (2020). Pandemia: os riscos da automedicação e os cuidados com a saúde. https://www.unisc.br/pt/noticias/pandemia-os-riscos-daautomedicacao-e-oscuidadoscom-a-saude 
https://portaleducacao.anapolis.go.gov.br/portal/pdf/Coronavirus-COVID19\%20\%20prefeitura\%20anapolis.pdf. Acesso em :13 de novembro de 202I Research, Society and Development, v. 10, n. 3, e58610313762, 2021(CC BY 4.o) | ISSN 25253409 | DOI: http://dx.doi.org/10.33448/rsd-vioi3.13762

Santos, M. F. F, Pereira, V. C. R, Guimarães Jr, P. R \& Lúcio N. M. P. (2020). Analysis of antimicrobial consumption in a community pharmacy in 2018. Research, Society and Development, 9(7), I-I5, e378974278.

SANTOS, Boaventura de Sousa. A Cruel Pedagogia do Vírus. Coimbra: Almedina, 2020. Souza, M. N.C. et. al., (202I). Ocorrência de Automedicação na população brasileira como estratégia preventiva para SARS-CoV-2. Pesquisa, Sociedade e

Desenvolvimento, io(I), e4451011933, 2021. I0.33448/rsd-vioir.II933.

WANG, M. et al. O remdesivir e a cloroquina inibem efetivamente o novo coronavírus recém-surgido (2019-nCoV) in vitro. Cell Res 30, 269-27I (2020). Disponível em:https://www.nature.com/articles/s41422-020-0282o?fbclid=IwAR3c5iygh65 XicnkrL6i6fJcWwioygNiLtI67SkcgREM4DyxxAcPauR uf5w\#citeas. Acesso em: is de novembro de 2021.

Conselho Federal de Farmácia - Brasil - Notícia: 30/04/2020 - Levantamento mostra como o medo da COVID-ı9 impactou venda de medicamentos [Internet]. Cff.org.br. 2020 [cited 27 May 2020]. Available from: http://www.cff.org.br/noticia.php?id=5747 